\title{
On the elasticity of phantom model networks with cyclic defects
}

\author{
Michael Lang* \\ Leibniz Institute of Polymer Research Dresden, Hohe Straße 6, 01069 Dresden, Germany \\ E-mail: lang@ipfdd.de
}

\begin{abstract}
The impact of finite cycles on the phantom modulus in an otherwise perfect network is computed exactly. It is shown that pending cycles reduce the phantom modulus of the network by $k T / V$ independent of junction functionality. The correction for nonpending cycles is larger than estimated previously within this particular approximation of the surrounding network structure. It is discussed that loop formation inevitably leads to streched chain conformations, if the loops are built step by step as part of the network structure. All network loops tend to contract simultaneously to optimize conformations, which leads to an increasing stretch of chains in larger loops that can be observed in computer simulations. Possible other corrections to phantom modulus that were left aside in previous work are discussed briefly.
\end{abstract}

Understanding the elasticity of a polymer network or gel is one of the key problems of polymer physics. Nowadays, it is generally accepted that rubber elasticity stems from two major contributions: the entanglement modulus, $G_{\mathrm{e}}$, caused by the non-cross-ability of the polymer strands and the phantom modulus, $G_{\mathrm{ph}}$, that resembles the contribution of network

${ }^{*}$ To whom correspondence should be addressed 
connectivity at the absence of entanglements. The exact solution of the phantom model of a perfect defect free model network containing no finite cyclic structures ("loops") is discussed in textbooks on polymer physics, see for instance, ref. 1 . It has been shown that linear and branched network defects can be treated by estimating the cycle $\operatorname{rank}^{2}$ of the network. But in contrast to the assumptions made in the textbook derivation, real polymer networks are made entirely of finite cycles, with a small average number of chains per cycle ${ }^{3}, 4$. Therefore, the generalization of our understanding of the phantom modulus from a perfect tree to a more realistic structure containing finite cycles is an open problem of polymer physics since the last 40 years $^{5}$.

Recently, first steps were made towards a solution of this problem $\underline{6}$ by considering the impact of isolated cyclic structures within an otherwise perfect tree ("ideal loop gas approximation", ILGA) made of $f$-functional junctions and ideal strands of $N$ segments between. Even though the ILGA is not realistic concerning the particular structure of the surrounding network, it should allow at least for a qualitative trend of the impact of finite cycles on elasticity. Quite interestingly, the results of ref. $\underline{6}$ were in clear disagreement to the result discussed in previous work ${ }^{2}$ or textbooks ${ }^{1}$. It was found that finite loops lead generally to a reduction of modulus. In particular, modulus was reduced by even more than $k T$ per pending cycle (cyclic structure with only a single connection to the rest of the network) for small junction functionality $f \leq 4$. If these results were correct, it would question whether the cycle rank is the correct concept to treat network defects at least for $f \leq 4$. And it would call for an improved estimate of the phantom modulus as proposed in ref. $\underline{6}$.

In the present paper, several problems related to the phantom modulus of a network containing finite cycles are addressed. Detailed computations are provided in the text document of the supporting information, while the main text provides an enhanced discussion of the results. The computation starts with the ILGA of ref $\underline{\underline{6}}$, which is solved here exactly for loops of arbitrary size. These results are compared with an alternate derivation considering explicitly the sequence of attaching strands to form a loop. It is shown that the process 
of network formation leads to stretched chain conformations once finite loops are formed inside the network by a step-wise addition of strands. These stretched conformations can be identified in simulation data providing evidence that cross-link fluctuations and contribution to modulus decouple for phantom networks made of finite cycles. Other mechanisms that might impact the phantom modulus are stressed briefly at the end of the manuscript.

The basic idea of the computations in section S1-S4 of the supporting informations is that the fluctuations of any part of the network around its average position in space can be modeled by the end-motion of a virtual chain that is attached to the non-fluctuating elastic background $(\mathrm{EB})$ on its other end ${ }^{7}$. The particular structure of the phantom network enters by introducing correlations among the fluctuations of connected molecules and for computing the size of the virtual chain that connects to EB.
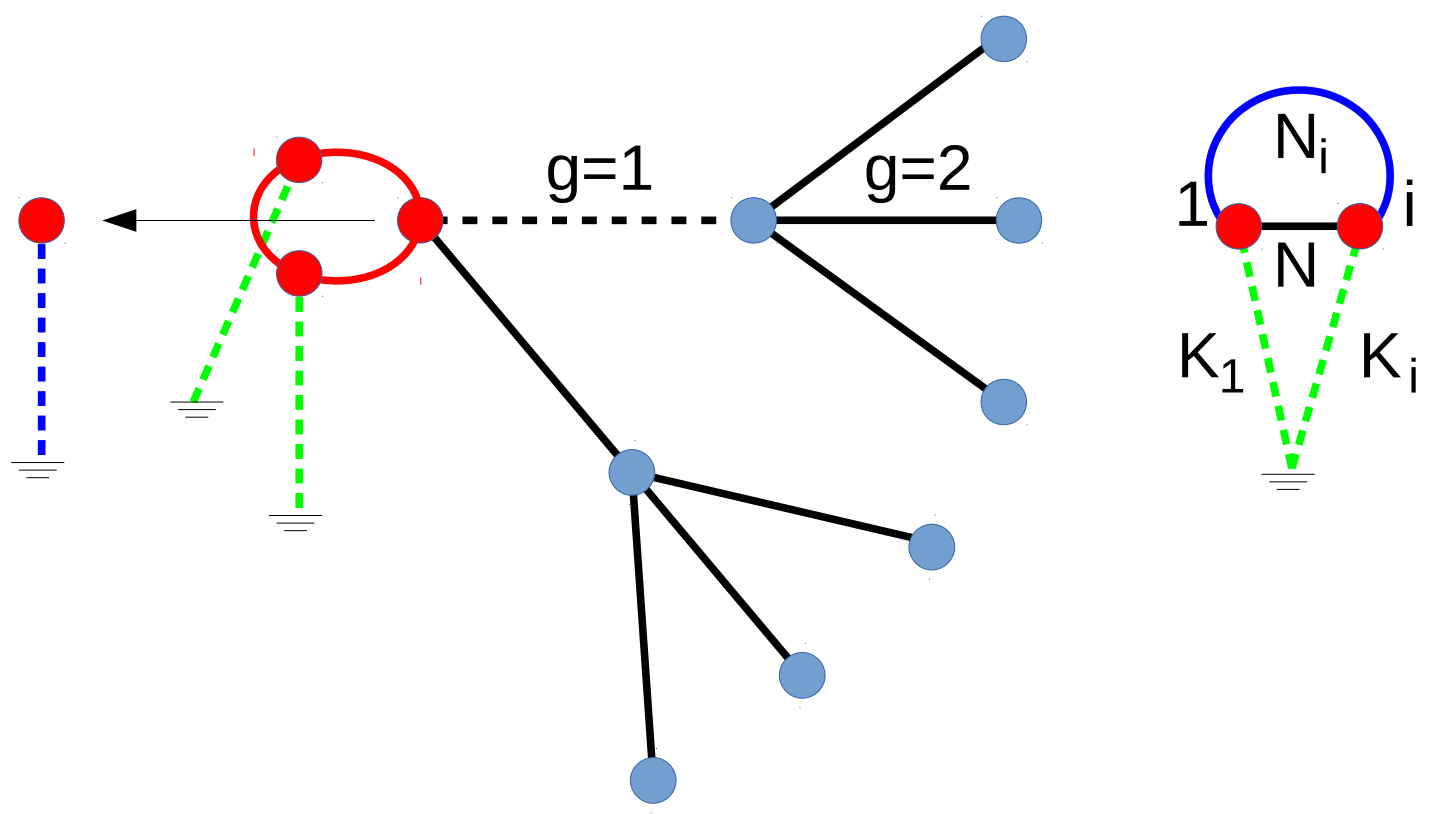

Figure 1: Left part: The fluctuations of each cross-link (red dots) inside the red loop are modeled by a virtual chain (blue dashed line) that connects to EB (ground symbol). The green dashed lines indicates the connections through the network to the EB outside of the loop. The first two generations of attached strands, $g=1$ and $g=2$, and the corresponding junctions on the way to EB (of the infinite tree to EB) are shown explicitly for one cross-link by black lines and gray dots. Right: reduced loop structure. One $N$-mer that connects junction 1 and $i$ directly, the strand $N_{\mathrm{i}}$ through the remainder of the loop but not through EB plus two strands $K_{1}$ and $K_{i}$ that connect junction 1 and $i$ through EB. 
We are interested in the elastic effectiveness $\epsilon$ of a strand in the network, which is defined here as the contribution of this strand to the shear modulus of the network in units of $k T / V$. Here $k$ is the Boltzmann constant, $T$ the absolute temperature and $V$ the volume of the sample. The computation of the effectiveness starts in section S1 of the supporting information with the general problem of the propagation of a distortion in junction fluctuations in an otherwise perfect network. To this end, it is summed over the modified elasticity of all attached strands in generation $g$ of connected strands away from the loop, see Figure 1 for illustration. Thus, the result of section S1 of the supporting information accounts for the impact of a loop on the surrounding network structure.

For computing the modified elastic effectiveness inside a loop made of $i>1$ elastic strands, we reduce the loop inside the network to the structure shown on the right of Figure 1) Then, there is one strand $N_{\mathrm{i}}$ that describes the connection between the first and last cross-link of the cycle that runs not through ground but through the rest of the finite cycle except of the $N$-mer, while the elastic strands $K_{1}$ and $K_{\mathrm{i}}$ describe the effective connection to ground after removing all cross-links except of cross-link 1 and $i$. The corresponding computations can be done exactly using $Y-\Delta$ transforms $^{\frac{9}{}}$ and are described in section $\mathrm{S} 2$ of the supporting information.

With these results, the change in elastic effectiveness caused by loops of arbitrary $i$ can be computed exactly within the ILGA. For $i=1$, the total reduction in elastic effectiveness per pending loop $(i=1)$ amounts to

$$
\Delta \epsilon_{i=1}=1
$$

independent of junction functionality $f$, see section S3 of the supporting information. Equation (1) agrees with the graph theory approach of cutting dangling loops ${ }^{2}$ and similar discussions in other works $\frac{10}{21}$, but is in contrast to ref. $\underline{\underline{6}}$ that obtained a different result for $f \leq 4$ 
For $i=2$, it is found in section S4 of the supporting information, that the net elastic effectiveness changes as

$$
\Delta \epsilon_{2}=\frac{2(f-1)}{f^{2}} .
$$

This result agrees with ref. $\cdot \underline{6}$ after considering the different normalization for elastic effectiveness and the coefficient of $2 / f$ for the difference in number density of network junctions and strands for all $f$.

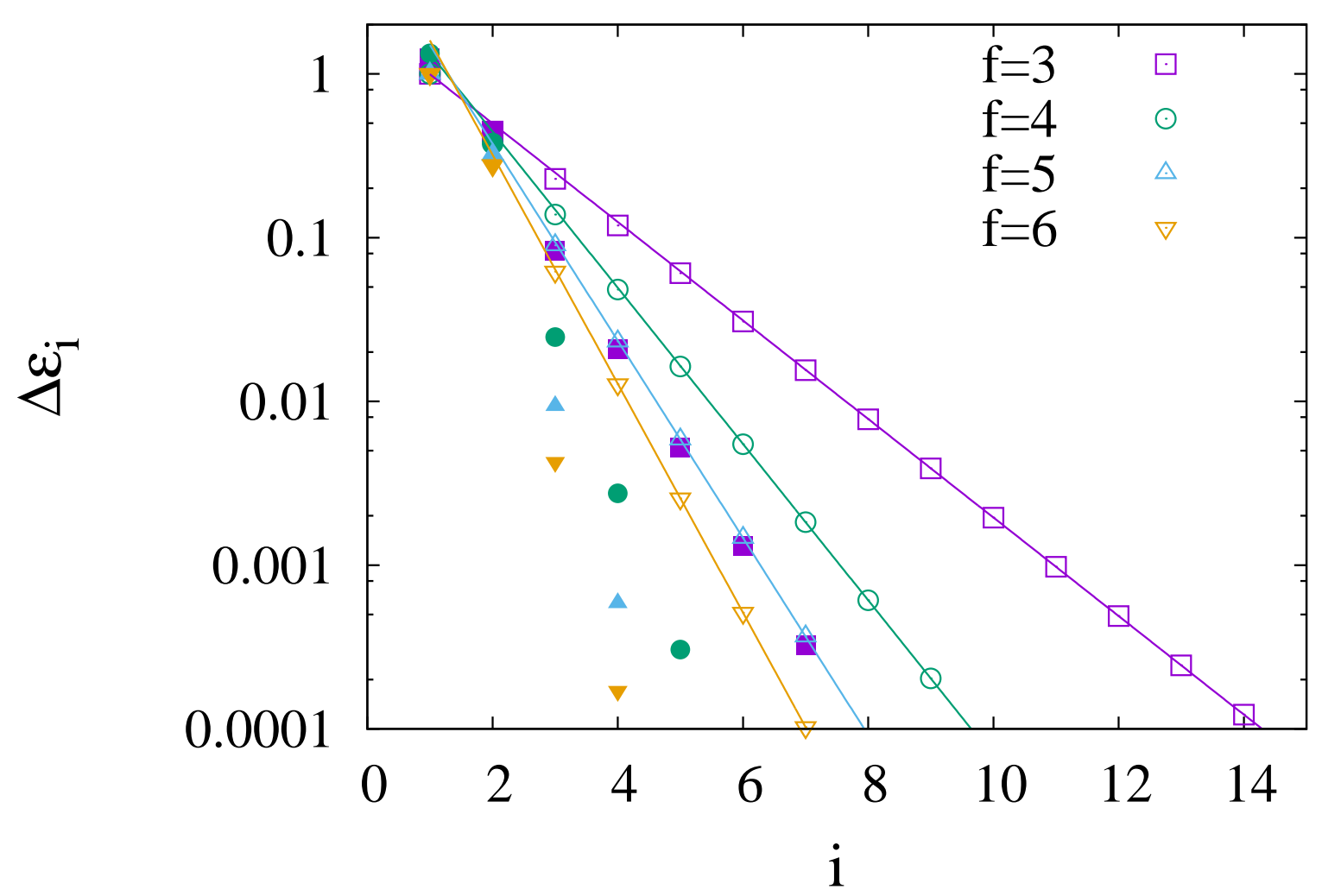

Figure 2: Reduction in elastic effectiveness $\Delta \epsilon_{i}$ as a function of $i$. Open symbols is computation of this work, full symbols is computation by ref. $\underline{6}$. The lines are a plot of equation (3).

Possible corrections due to finite loops were computed numerically for $i>2$ and compared with the approximation of ref. $\underline{6}$ in Figure 2. The exact solution approaches for large $i$ quickly the approximation

$$
\Delta \epsilon_{\mathrm{i}} \approx \frac{2(f-2)}{(f-1)^{i}},
$$


that was included as lines in Figure 2, see section S4 of the supporting information for more details. The exact solution of the present work is clearly larger than the approximate result derived previously $\underline{\underline{6}}$, see Figure 2 , but remains a small correction for large $i$. This difference compensates to a large portion the over-estimate of the pending loops for $f \leq 4$ in previous work ${ }^{\underline{6}}$.

The above equations allow for a quantitative test of the impact of finite loops on the elasticity of a network, if combined with statistical information on the frequency of these finite loops ${ }^{12-14}$. But as discussed below, finite loops are neither the only important correction for the elasticity of model networks nor must the above results provide a correct estimate for the corrections due to finite loops in any situation.

The weak point of the above results is that the derivation relies solely on a consideration of cross-link fluctuations and how these change when inserting a finite loop. As discussed in section S5 of the supporting information, such an approach is fully legitimate when linking instantaneously chains to a tree-like network when the ensemble average chain conformations are in reference state. Then, the conformations of the network chains can be split into a time average part and a fluctuating part, whereby the latter is solely related to cross-link fluctuations and the former to the time average stretch of the chains. The portion by which this time average conformations are shorter than the square reference chain size $R_{0}^{2}$ is identified as the elastic effectiveness $\epsilon$ and determines the contribution to modulus in multiples of $k T / V$.

However, such a line of arguments fails when considering finite loops. The intricate point is that the ensemble average conformations of a chain as part of a loop differ from the conformations of a linear strand: the unperturbed average square size between two adjacent "cross-links" along an isolated loop of $i$ chains is equivalent to the unperturbed size of a linear strand with

$$
N_{\mathrm{a}}=N \frac{i-1}{i}
$$

segments. Thus, whenever a loop is built chain by chain as part of a network (see section 
S6 of the supporting information for more details), these strands enter the loop with unperturbed size but tend to contract once the loop is closed to a square size that is smaller by a factor of $(i-1) / i$. This creates extra stress on the surrounding network chains that is not recognized when considering or measuring fluctuations, since the fluctuations depend solely on connectivity (for a network of Gaussian chains) and not on the spatial extension of the combined strands. The contribution to modulus of a particular strand, however, is proportional to the spatial extension of the time average strand, see section S5 of the supporting information, and thus, proportional to the extension of the corresponding combined strand for a given $N$.

What complicates the discussion is that there is an alternative route how a loop can become part of the network: if a loop is closed prior to attaching it at least twice to the network, it can relax to the average loop size. This latter route is important for small $i$ and close to the gel point, while the former process dominates towards the end of the reactions. Both ways of attaching strands lead to a different contribution to elastic modulus, see section S6 of the supporting information. But only the latter route agrees with the results that were derived from considering cross-link fluctuations.

A second intricate point of randomly connected polymer networks is that the chains inside a network are simultaneously part of several loops, whereby the corresponding frequency distribution for participation is rather broad, see Figure 5 of ref. $\underline{15}$ for an example. Thus, the chains inside the network cannot accommodate all of the constraints arising from the connected loops simultaneously. But one could expect that the impact of the smallest loop where a chain is part of stands out, since $N-N_{\mathrm{a}}$ is largest for the smallest loops. Thus, the number of strands $i_{\min }$ of the smallest loop where a network strand is part of needs to be determined. The distribution of this $i_{\text {min }}$ is typically peaked at a small $i_{\text {min }}$ in the range of 6-8 for end-linked model networks with $f=4$ and an overlap number of $\approx 8$ of an end-linked network, see section S7 of the supporting information. Note that some more information on loop size distributions for networks with different structure and the corresponding peak 
position is available in ref. $\underline{\underline{4}}$.

The $i_{\text {min }}$ was determined for an untangled network where the time average conformations of the chains have been analyzed previously $\underline{\underline{16}}, \underline{17}$. Figure 3 compares the square ratios of instantaneous chain size, $R$, time average chain size, $R_{\mathrm{t}}$, and reference chain size in melt, $R_{0}$. Averages over the whole network are included at $i_{\min }=0$.

The average chain size in the network appears to be almost not stretched because of $\left(R / R_{0}\right)^{2} \approx 1.03$, however, this observation is partially biased by the contribution of pending loops. The fluctuating chain size in non-pending loops $i>1$ is stretched by $\approx 8 \%$ as compared to the unperturbed size in melt. For this particular sample, this correction is therefore larger than the impact of pending loops that is here a correction of $\approx 5 \%$, see section S7 of the supporting information. Now, let us ignore this stretching correction and consider only the normalized time average size of all network strands with $2\left(R_{t} / R_{0}\right) \approx 0.74$. Consideration of conversion of $p=0.95$ leads to an estimate of $2\left(R_{t} / R_{0}\right) \approx 0.8$, which is further reduced by the $5 \%$ of pending loops to $2\left(R_{t} / R_{0}\right) \approx 0.75$. If compared with a measurement of modulus, one would probably conclude that a consideration of conversion and pending loops is sufficient to describe the data. But the stretched chain conformations and impact of finite loops with $i>1$ would be missed by this simplified analysis.

A more detailed view is possible when looking into the dependence of these ratios as a function of $i_{\min }$ that indicate a striking dependence on the loop size. Quite interestingly, when dividing out stretch by normalizing time average size with respect to fluctuating size, the corresponding ratio $2\left(R_{t} / R\right)$ approaches roughly equation (4). This observation can be interpreted such that all cyclic structures of the network attempt to maximize entropy simultaneously by putting the surrounding chains and loops under tension. Thus, indeed, one could consider an "ideal gas of loops" as a reference state for the network. But this ideal gas is under tension and one has to understand how this tension enters the system or is being relaxed during the process of network formation along with the development of the size distribution of loops as a function of conversion. 


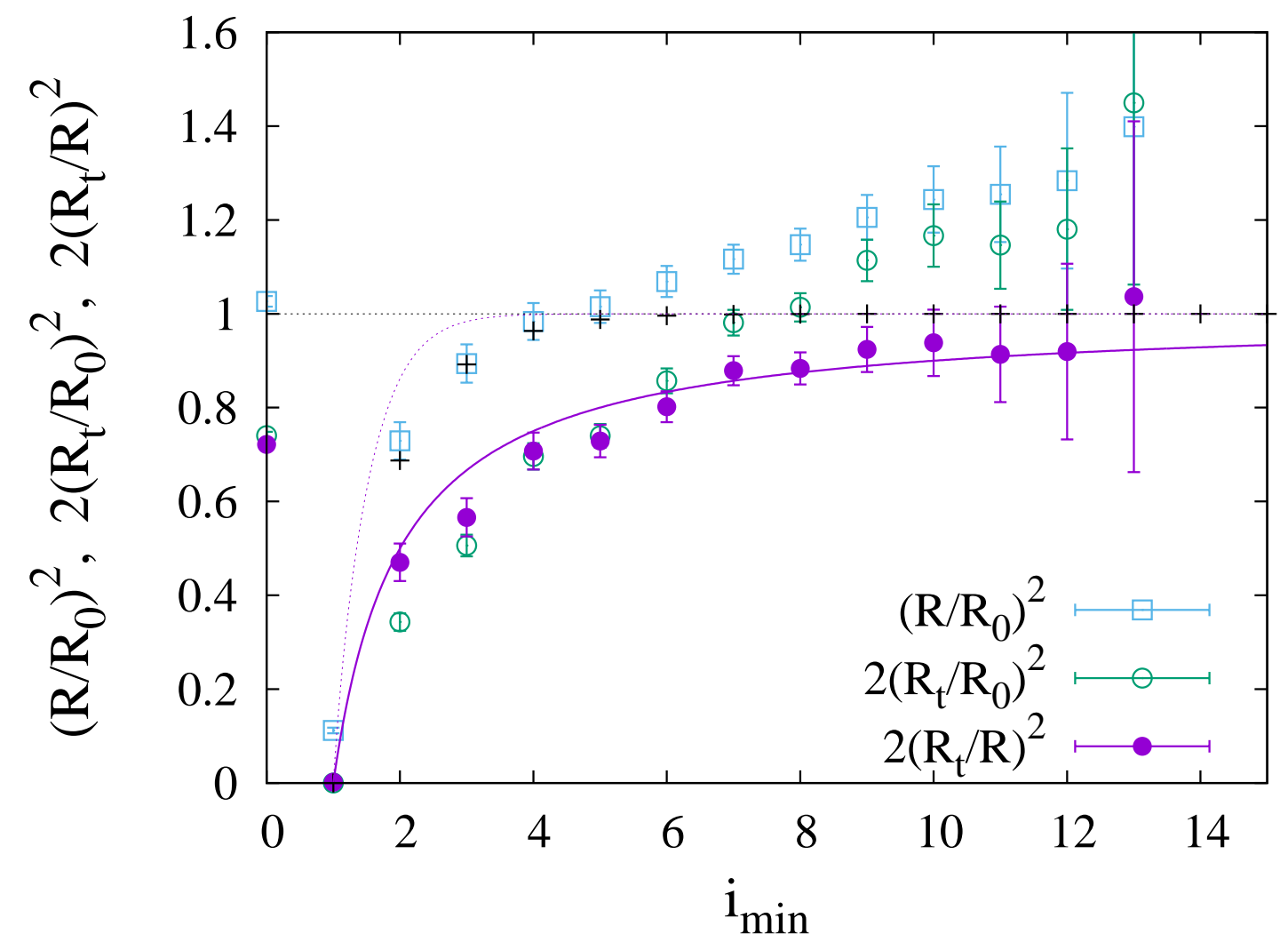

Figure 3: Normalized instantaneous square size of active chains, $\left(R / R_{0}\right)^{2}$, twice the normalized time average square size of active network chains $2\left(R_{t} / R_{0}\right)^{2}$, and twice the ratio of time average and instantaneous size $2\left(R_{t} / R\right)^{2}$ as a function of $i_{\text {min }}$. The black crosses indicate the estimated instantaneous chain size when considering the ILGA as discussed in the supporting information. The averages over all $i_{\text {min }}$ are included as extra data points at $i_{\text {min }}=0$. The continuous line is equation (4), the red dashed line results from equation (11) of ref. $\underline{6}$ that was considered as an approximation for $i \geq 3$. 
In this context, it has to be mentioned that linking chains from non-reference chain conformations has been discussed previously in the case of entangled networks ${ }^{8}$, but similar arguments apply for phantom networks. The key idea is that towards full conversion, possible reaction partners are predominantly found at a distance larger than reference size, since otherwise, these reaction partners would have been encountered previously by the compact exploration of space of reactive groups attached to polymers $\frac{18}{}$ for diffusion controlled reactions. This leads to an extra stretch of the network strands as a function of conversion. Note that also for reaction controlled systems, a part of this bias towards more extended chain conformations must survive. Thus, a thorough investigation of the phantom modulus of random model networks needs to separate this conversion dependent stretch from the cycle dependent part and should address the rate dependence of reactions and relaxation processes. This is clearly beyond the scope of the present letter that aims to point out the main problems that have to be addressed.

Beyond the above problem of deformed chain conformations in a polymer network, there are additional points that require more attention in future work. First, these model networks are typically prepared by a co-polymerization reaction. Composition fluctuations of the reacting species freeze in beyond the gel point and may lead to a systematic shift of the data away from a perfect mixing assumed in theory as discussed previously ${ }^{19}$. Second, full conversion is never reached but phantom modulus was not corrected for incomplete conversion in ref. $\underline{6}$. Note that earlier work on the network disassembly spectrometry recognized a measurable amount of pending chains ${ }^{20}$ hinting in this direction. Finally, the above results based upon the ILGA cannot rule out that there are additional cooperative effects among connected loops, since real networks are made entirely of finite loops. For these, we have no concepts right at hand, but an analysis of the elastic properties of network strands similar to Figure 3 might lead to an identification of such effects. 


\section{Acknowledgments}

The author acknowledges support from the DFG (grant LA2735/2-2) and helpful discussions with A. Sharma, T. Müller, T. Kreer, K. Suresh Kumar, and J.-U. Sommer.

\section{Associated Content}

The supporting information contains a FORTRAN code in a separate file that performs the computations as described in the supporting information. The seven sections of the text document in the supporting information concern: 1) The propagation of a network distortion and the corresponding modification in elastic effectiveness of the surrounding network chains. 2) The reduction of non-pending loops of arbitrary number of chains to loops containing two chains. 3) The elastic effectiveness of pending cycles. 4) The elastic effectiveness of non-pending cycles as derived from considering cross-link fluctuations only. 5) A simplified discussion of the classical phantom model and how the cross-linking process is taken into account. 6) The problem of how a loop is incorporated into the network, which can cause elastic contributions different to the ones estimated from cross-link fluctuations. 7) A test of principles by comparing with preliminary simulation data.

\section{References}

(1) Rubinstein, M.; Colby, R. H. Polymer Physics; Oxford University Press, 2003.

(2) Flory, P. J. Elastic activity of imperfect networks. Macromolecules 1982, 15, 99-100.

(3) Lang, M.; Michalke, W.; Kreitmeier, S. A statistical model for the length distribution of meshes in a polymer network. J. Chem. Phys. 2001, 114, 7627-7632.

(4) Lang, M.; Kreitmeier, S.; Göritz, D. Trapped Entanglements in Polymer Networks. Rubber Chem. Tech. 2007, 80, 873-894. 
(5) Flory, P.; Gordon, M.; McCrum, N. Statistical thermodynamics of random networks. Proc. R. Soc. A 1976, 351, 351-380.

(6) Zhong, M.; Wang, R.; Kawamoto, K.; Olsen, B. D.; Johnson, J. A. Quantifying the impact of molecular defects on polymer network elasticity. Science 2016, 353, 12641268.

(7) Rubinstein, M.; Panyukov, S. Elasticity of polymer networks. Macromolecules 2002, 35, 6670-6686.

(8) Lang, M. Relation between Cross-Link Fluctuations and Elasticity in Entangled Polymer Networks. Macromolecules 2017, 50, 2547-2555.

(9) Kenelly, A. E. Equivalence of triangles and three-pointed stars in conducting networks. Elect. World 1899, 34, 413-414.

(10) Dusek, K.; Gordon, M.; Ross-Murphy, S. B. Graphlike state of matter. 10. Cyclization and concentration of elastically active network chains in polymer networks. Macromolecules 1978, 11, 236-245.

(11) Panyukov, S.; Rabin, Y. Statistical physics of polymer gels. Phys. Rep. 1996, 269, 1-131.

(12) Suematsu, K. Recent progress in gel theory: ring, excluded volume, and dimension. Adv. Polym. Sci. 2002, 156, 137-214.

(13) Lang, M.; Göritz, D.; Kreitmeier, S. Intramolecular reactions in randomly end-linked polymer networks. and linear (co)polymerizations. Macromolecules 2005, 38, 25152523.

(14) Wang, R.; Alexander-Katz, A.; Johnson, J. A.; Olsen, B. D. Universal cyclic topology in polymer networks. Phys. Rev. Lett. 2016, 116, 188302. 
(15) Lang, M.; Michalke, W.; Kreitmeier, S. Analysis of trapped entanglements in polymer networks. J. Comp. Phys. 2003, 185, 549-561.

(16) Lang, M.; Sommer, J.-U. Analysis of Entanglement Length and Segmental Order Parameter in Polymer Networks. Phys. Rev. Lett. 2010, 104, 177801.

(17) Lang, M. Monomer fluctuations and the distribution of residual bond orientations in polymer networks. Macromolecules 2013, 46, 9782-9797.

(18) de Gennes, P. G. Kinetics of diffusioncontrolled processes in dense polymer systems. I. Nonentangled regimes J. Chem. Phys. 1982, 76, 3316-3321.

(19) Lang, M.; Schwenke, K.; Sommer, J.-U. Short cyclic structures in polymer model networks: A test of mean field approximation by monte carlo simulations. Macromolecules 2012, 45, 4886-4895.

(20) Zhou, H.; Woo, J.; Cok, A. M.; Wang, M.; Olsen, B. D.; Johnson, J. A. Counting primary loops in polymer gels. PNAS 2012, 109, 19119-19124. 

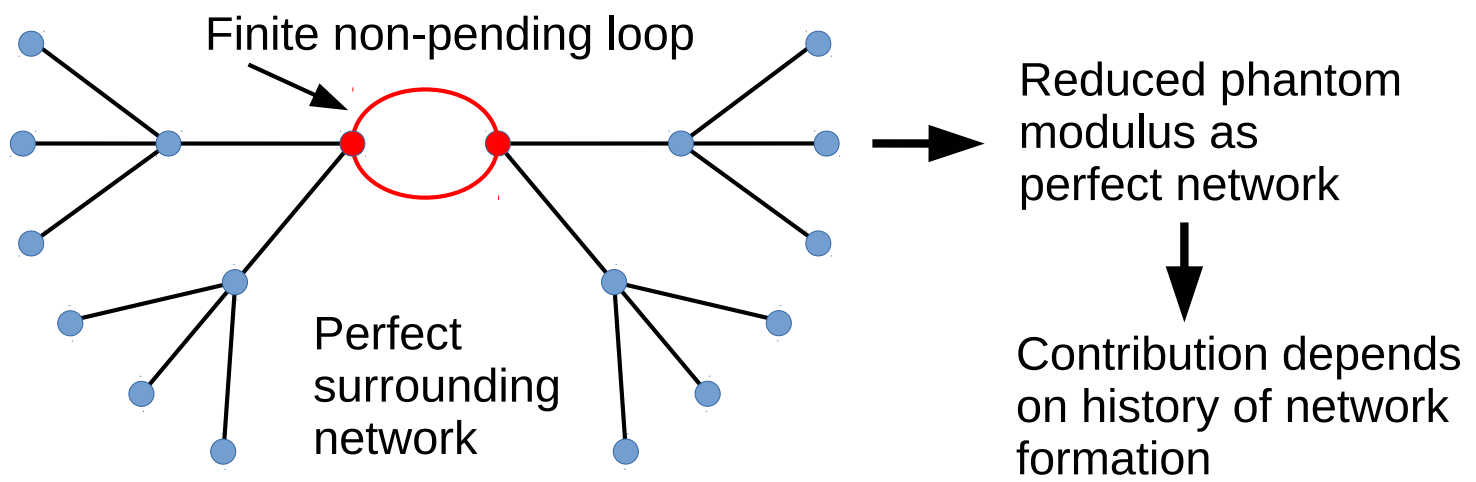